\title{
CARACTERIZACIÓN DEL CONSUMO ELÉCTRICO EN LAS GRANJAS DE VACUNO LECHERO DE CASTILLA Y LEÓN
}

\author{
DAIRY CATTLE FARM ELECTRICITY CONSUMPTION IN CASTILLA Y LEÓN \\ Bartolomé, D.J.*; Posado, R.; Bodas, R.; Tabernero de Paz, M.J.; García, J.J. \\ y Olmedo, S.
}

Instituto Tecnológico Agrario. Subdirección de Investigación y Tecnología. Consejería de Agricultura y Ganadería. Junta de Castilla y León. Finca Zamadueñas. Valladolid. España. *ita-barrodda@itacyl.es

\section{Palabras clave adicionales}

Ahorro. Eficiencia energética. Ordeño. Sostenibilidad.

\section{RESUMEN}

Con el objetivo de conocer el consumo de electricidad y su distribución en las explotaciones de ganado vacuno lechero de Castilla y León se realizaron auditorías energéticas a 80 explotaciones, recogiéndose datos sobre sus características, equipos y tiempos de utilización (sala de ordeño, refrigeración, limpieza, agua caliente, ventilación, otros dispositivos) y factura eléctrica. A partir de estos datos se calcularon los índices de consumo medio anual y los índices de utilización de electricidad por vaca productora y por litro de leche producido. Los equipos que más electricidad consumen son la bomba de vacío $(8948 \mathrm{kWh}$ año) y el tanque de frío ( $6030 \mathrm{kWh} / a n ̃ o)$, seguidos por los sistemas de limpieza ( $5244 \mathrm{kWh} / a n ̃ o)$. Los sistemas de ordeño en espina de pescado mostraron un consumo inferior $(7 \mathrm{kWh})$ al de los robots de ordeño (24 kWh) o las salas rotativas ( $26 \mathrm{kWh}$ ). El consumo eléctrico anual medio se sitúa en los $516 \mathrm{kWh}$ por vaca productora o $51 \mathrm{kWh}$ por cada $1000 \mathrm{~kg}$ de leche producida. La optimización del consumo eléctrico en las explotaciones de ganado vacuno lechero es posible, pero pasa por la evaluación del consumo real producido en cada equipo y proceso de la explotación.

\section{SUMMARY}

Eighty energy audits were carried out to 80 dairy cattle farms in Castilla y León, in order to determine electricity consumption and its distribution. Data regarding their characteristics, equipment (milking, cooling, cleaning, hot water,

\author{
Additional KEYWORDS \\ Energy efficiency. Milking. Saving. Sustainability.
}

ventilation and other devices), usage times and electricity bills were collected. From these data, average electricity annual consumption and utilization rates per cow and per litre of produced milk were calculated. Vacuum pump (8948 kWh/ year) and the cooling tank (6030 kWh/year) were the most consumptive equipments, followed by cleaning systems (5244 kWh/year). Herringbone milking parlours showed lower consumption (7 $\mathrm{kWh})$ than milking robots (24 kWh) and rotary milking parlours (26 kWh). Average electricity consumption stands at $516 \mathrm{kWh}$ per productive cow and year or $51 \mathrm{kWh}$ per $1000 \mathrm{~kg}$ of milk produced per year. The optimization of power consumption in the dairy cattle farms is possible, but passes through the evaluation of actual consumption produced in each team and exploitation process.

\section{INTRODUCCIÓN}

En los últimos 10 años las explotaciones de ganado vacuno de leche de España han doblado su tamaño, pasando de tener una media de 16 animales por explotación en 1999 (19 para Castilla y León) a 31 en 2009 (44 en Castilla y León), según los datos del último censo agrario disponible (INE, 2002; INE, 2012; Jimeno y Castro, 2009). El aumento en el tamaño de las explotaciones ha ido unido a una utilización cada vez mayor de equipos automatizados con el fin de hacer- 


\section{BARTOLOMÉ, POSADO, BODAS, TABERNERO DE PAZ, GARCÍA Y OLMEDO}

las más competitivas, ofertar empleos en condiciones laborales equiparables a otros sectores y suplir la carencia de mano de obra disponible. Estas circunstancias han determinado un aumento gradual del consumo de energía en estas explotaciones (Ludington y Johnson, 2003).

Independientemente del incremento en el consumo de electricidad, existen procesos en los que éste podría reducirse o hacerse económica y energéticamente más eficiente (Upton et al., 2010). Además, hay que tener en cuenta que el incremento en los precios de la electricidad repercute negativamente sobre el balance económico de las explotaciones, reduciendo sus beneficios e ingresos netos (Murgia et al., 2008). En este sentido, sería deseable el establecimiento de prácticas de manejo y medidas de ahorro para realizar un uso más racional de la energía, promover el ahorro e incrementar la eficiencia energética de las explotaciones. No obstante, antes de realizar estas recomendaciones, es necesario conocer los patrones de consumo, para saber dónde, cómo y en qué momentos se produce éste.

El considerable aumento del consumo de electricidad en este tipo de explotaciones en los últimos años, y sus implicaciones económicas y ambientales, han promovido la caracterización del mismo en todas las zonas lecheras, si bien los estudios encontrados en la bibliografía son más bien escasos y con resultados muy divergentes. No obstante, conviene recordar la variabilidad entre sistemas de producción, tipos y características del equipamiento de las explotaciones así como las condiciones edafoclimáticas de las zonas de ubicación, que determinan que los índices de utilización de la energía varíen considerablemente, no sólo de unas zonas a otras, sino entre granjas relativamente próximas. Así, en los diferentes países donde se han realizado estudios de este tipo proporcionan un amplio rango de datos; por ejemplo el consumo eléctrico anual estimado por vaca en Estados Unidos oscila entre los 700 y $1200 \mathrm{kWh}$, dependien- do de los estudios (Ludington y Johnson, 2003; WDATCP, 2006), en Irlanda es de alrededor de $300 \mathrm{kWh}$ (Upton et al., 2010), en Nueva Zelanda es de $163 \mathrm{kWh}$ (Hartman y Sims, 2006), en Italia de 466 kWh (Murgia et al., 2008), en el Reino Unido de 330 (Trimble, 2009) y en Francia de $470 \mathrm{kWh}$ (Dollé, 2007; Béguin et al., 2008). Aunque las diferencias entre los distintos estudios son elevadas, incluso dentro de cada estudio, la cantidad de energía necesaria en las granjas varía ampliamente en función del tamaño del rebaño, el nivel de tecnología empleado y el tipo de manejo. Así, en algunos casos, el máximo valor observado en las mediciones es el doble que el mínimo (Hartman y Sims, 2006; Dollé, 2007; Murgia et al., 2008). Por otra parte, conviene destacar que, si bien la idea general de todos los estudios es la misma, el número y tipo de granjas encuestadas, el periodo considerado y los procesos tenidos en cuenta varían de unas a otras. En este sentido, los estudios más exhaustivos son los relativos a Italia (Murgia et al., 2008) y Francia (Dollé, 2007; Béguin et al., 2008). Los estudios de Hartman y Sims (2006) y Upton et al. (2010), que tomaron datos en 62 y 3 granjas, respectivamente, no hacen mención expresa al manejo de estiércol o la ventilación (Hartman y Sims, 2006, tampoco consideran la iluminación), mientras que el estudio de Trimble (2009) no menciona procesos concretos, pero sí especifica que de las 100 granjas auditadas se han eliminado aquellas con robot de ordeño (por su elevado consumo). En cualquier caso, los indicadores de eficiencia energética no parecen estar relacionados con el tamaño del rebaño, existiendo siempre un amplio margen para seguir optimizando el consumo (Murgia et al., 2008).

Las auditorías energéticas se pueden definir como un proceso sistemático mediante el cual se obtiene un conocimiento suficientemente fiable del consumo energético de una explotación y se detectan los factores que afectan al mismo. Deben recogerse, asimismo, datos relativos a otras ca- 


\section{CONSUMO ELÉCTRICO EN GRANJAS DE VACUNO LECHERO}

racterísticas de la explotación que permitan definir indicadores de consumo en términos de productividad (número de vacas en lactación, número de litros producidos, etc.). El objetivo final será identificar, evaluar y ordenar las distintas oportunidades de ahorro en función de su rentabilidad económica (IDAE, 2010; Murgia et al., 2008).

Desde el punto de vista socioeconómico, la importancia del sector del vacuno de leche en Castilla y León resulta evidente, ya que esta comunidad autónoma ocupaba en 2011 el primer lugar a nivel nacional en censo de ganado vacuno (más de 95 mil vacas de ordeño) y el segundo, después de Galicia, por producción de leche de vaca (775 mil toneladas, aproximadamente un 13\% de la cuota nacional) (MARM, 2010). Por otra parte, las explotaciones de vacuno de leche emplean principalmente mano de obra familiar y están asentadas en zonas rurales de tradición lechera, contribuyendo así a fijar población en lugares donde las alternativas a esta actividad habitualmente son escasas. En este sentido, un aumento en la eficiencia energética de las explotaciones promoverá un uso más sostenible de los recursos disponibles que, en último término, se traducirá en una mejora de la productividad.

El objetivo del presente trabajo es, por tanto, conocer el consumo de electricidad de las explotaciones de vacuno de leche de Castilla y León.

\section{MATERIAL Y MÉTODOS}

Para llevar a cabo este estudio se han realizado auditorías energéticas a 80 explotaciones de vacuno de leche de raza Holstein durante el año 2011 ubicadas en cuatro provincias de Castilla y León: Ávila, Palencia, Valladolid y Zamora. Se seleccionaron estas provincias por ser las de mayor censo y producción de la comunidad, clasificándose sus explotaciones en función de la cuota de producción láctea que tenían asignada y procurando una distribución homogénea entre tramos de producción. Así, 25 de las explotaciones seleccionadas tenían una cuota asignada de entre 100000 y $200000 \mathrm{~kg}$, 26 explotaciones tenían entre 200000 y $400000 \mathrm{~kg}$, mientras que 29 explotaciones tenían una asignación de cuota superior a los $400000 \mathrm{~kg}$. Por razones técnicas no se tuvieron en cuenta aquellas explotaciones cuya cuota de producción de leche era inferior a $100000 \mathrm{~kg}$.

Las auditorías, cuyo diseño se realizó teniendo en cuenta los objetivos previstos y conforme a las recomendaciones de la bibliografía(IDAE, 2010; Murgia et al., 2008), se estructuraron para recoger la siguiente información correspondiente a los 12 meses previos a la auditoría:

Características de la explotación: producción lechera, cuota láctea asignada, número de animales, número de vacas en lactación.

Sala de ordeño: tipo, duración y horario de ordeño y de limpieza.

Bombas de vacío, bombas de leche $y$ bombas de agua: tipo, potencia, horas de funcionamiento, presencia de variadores de frecuencia.

Tanques de frío: número, tipo, situación, capacidad, potencia, horas de funcionamiento.

Iluminación: tipo de lámparas, número, potencia, horas y horario de funcionamiento.

Motores de limpieza: tipo, potencia, horas de funcionamiento.

Termo eléctrico: tipo, capacidad, potencia, temperatura y volumen de consumo de agua caliente.

Ventilación: número de ventiladores, potencia, temporadas y horas de funcionamiento.

Otros motores o dispositivos eléctricos: tipos, potencias, horas de funcionamiento.

Adecuado mantenimiento y limpieza de todos los sistemas: obtención, conducción y enfriamiento de la leche, aislamiento de tuberías, mantenimiento preventivo o pe- 


\section{BARTOLOMÉ, POSADO, BODAS, TABERNERO DE PAZ, GARCÍA Y OLMEDO}

riódico de todos los equipos.

Detalles de la factura eléctrica: potencia contratada, potencia demandada, tarifa, exceso de potencia, energía activa y reactiva consumida, alquiler de equipos de medida, impuestos.

Toda la información recogida fue introducida en una base de datos, a partir de los cuales se calcularon los índices medios de consumo anual que se muestran en los resultados: tanque de frío, bomba de vacío, iluminación, ventilación, termo eléctrico, cintas de limpieza, otros (engloba procesos tales como el bombeo de agua o la distribución de alimento, entre otros).

A partir de estos cálculos, se estimó la cantidad de energía eléctrica asociada a los siguientes procesos: ordeño (bombas de vacío y bomba de la leche), limpieza y desinfección (cintas de limpieza, arrastraderas, hidrolimpiadoras) y enfriamiento de la leche (tanque de frío).

Para los cálculos económicos se han utilizado los precios de la electricidad (1 $\mathrm{kWh}=0,13$ euros $)$ y de la leche $(1 \mathrm{~kg}=0,319$ euros) en el momento de la realización del presente estudio (enero de 2011), disponibles en la página web del Ministerio de Industria, Energía y Turismo (www.minetur. gob.es) y del Ministerio de Agricultura, Alimentación y Medio Ambiente (www. magrama.gob.es), respectivamente.

Se calcularon los índices de utilización de la electricidad por vaca productora y por unidad de leche producida (Edens et al., 2003; Ludington y Johnson, 2003; Murgia et al., 2008).

Los valores medios de consumo y gasto y su error estándar se calcularon teniendo en cuenta los datos disponibles para cada parámetro. Asimismo los datos de los consumos anuales y medios, así como los costes anuales se sometieron a un análisis de varianza de una vía, para estudiar los efectos del tipo de sala de ordeño, comparando solamente las salas en espina de pescado $(n=48)$, rotativa $(n=5)$ y robot de ordeño $(n=5)$. Estos mismos datos se analizaron de forma similar para estudiar el efecto del nivel de producción $(<200000 \mathrm{~kg}$ leche/año, $200000-400000 \mathrm{~kg}$ leche/ año y $>400000 \mathrm{~kg}$ leche/año).

\section{RESULTADOSYDISCUSIÓN}

Como se describe en el apartado anterior, la población muestral de explotaciones encuestadas cubría un amplio rango de producciones y tipologías de granja. No obstante, el tipo medio de explotación encuestado vendría definido por una granja que tiene $77( \pm 8,5)$ animales en producción y 57 $( \pm 6,4)$ en cría, y que produce algo más de $800000 \mathrm{~kg}$ de leche al año $(2293 \pm 275,0 \mathrm{~kg} /$ día).

Los datos medios de consumo de energía eléctrica para cada equipo así como el impacto económico que pueden suponer, estimados a partir de su potencia y tiempo de funcionamiento, aparecen recogidos en la tabla I. La contribución que cada uno de estos equipos representa respecto al consumo total puede verse en la figura 1. Respecto a los equipos que están presentes en todas las explotaciones, la bomba de vacío (que funciona al menos dos veces al día de forma continua por un periodo nunca inferior a 1 hora) es el equipo que presenta un

Tabla I. Consumo eléctrico estimado (en $k W h$ y euros/año) de los principales equipos de una explotación de ganado vacuno lechero. (Estimated electricity consumption (in kWh and euros/year) of the main equipment in a dairy cattle farm).

\begin{tabular}{lcccc}
\hline & \multicolumn{2}{c}{$\mathrm{kWh} / \mathrm{año}$} & \multicolumn{2}{c}{ euros/año } \\
& media & e.e.m. & media & e.e.m. \\
\hline Tanque de frío & 6030 & 606,2 & 784 & 78,8 \\
Bomba de vacío & 8984 & 1199,1 & 1168 & 155,9 \\
lluminación & 1182 & 189,7 & 154 & 24,7 \\
Ventilación & 1476 & 377,1 & 192 & 49,0 \\
Termo eléctrico & 1032 & 67,8 & 134 & 8,8 \\
Cintas de limpieza & 5244 & 2810,1 & 682 & 365,3 \\
Otros & 3466 & 637,1 & 451 & 82,8 \\
\hline
\end{tabular}




\section{CONSUMO ELÉCTRICO EN GRANJAS DE VACUNO LECHERO}

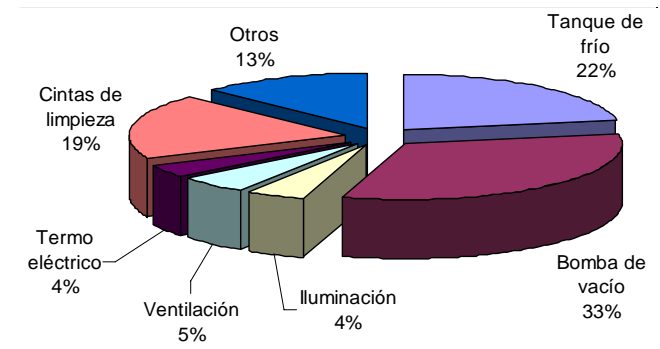

Figura 1. Distribución del consumo eléctrico entre los diferentes equipos de una explotación de ganado vacuno lechero. (Distribution of electricity consumption among different equipment in a dairy cattle farm).

mayor consumo de electricidad ( $8948 \mathrm{kWh} /$ año). Lógicamente, el siguiente equipo por orden de consumo es el tanque de frío, que debe bajar la temperatura de la leche de forma rápida y mantenerla hasta su posterior recogida y transporte o procesado (6030 kWh/año). Otro de los elementos necesarios en las explotaciones son los dispositivos de calentamiento de agua. Pese a su importancia, el peso que representan en el consumo eléctrico total de la explotación es relativamente pequeño (menos del $4 \%$ del consumo total de electricidad, figura 1 ). Este hecho puede venir motivado, al menos en parte, porque casi un $40 \%$ de las explotaciones auditadas calentaban el agua con calderas de gas natural o gasoil, lo que disminuye la importancia relativa del calentador eléctrico. No obstante, considerando solamente aquellas granjas que utilizaban termo eléctrico, éste no supuso más que el $6 \%$ del consumo total de electricidad. Por último, el gasto eléctrico en iluminación, presente en todas las granjas, no representa más del 4,3\% (figura 1) del consumo total (1182 kWh/año).

En relación a los equipos que pueden o no estar presentes en las granjas, un $40 \%$ de éstas poseían arrobaderas, arrastraderas o cintas de limpieza. Éstos equipos suponen un consumo de $5244 \mathrm{kWh}$ al año, seguidas en orden de importancia de consumo por los sistemas de ventilación (presentes en un $24 \%$ de las explotaciones), los cuales suponen algo más del $5 \%$ del consumo de electricidad (figura 1).

Una vez conocido el consumo eléctrico de cada equipo, puede estimarse el consumo de cada uno de los tres principales procesos de producción en una explotación de vacuno de leche: ordeño $(12,6 \mathrm{kWh})$, enfriamiento de la leche $(8,3 \mathrm{kWh})$ y tareas de limpieza y desinfección $(6,4$ kWh). Así, considerando la frecuencia con que se realizan estos procesos (2 veces al día, cada día), se puede estimar el consumo eléctrico anual que representa cada proceso (tabla II). Por consiguiente, el proceso más costoso, desde el punto de vista energético, es el ordeño, seguido por el enfriamiento de la leche y por la limpieza y desinfección.

No obstante, para poder establecer una comparación entre diferentes granjas, resulta interesante estimar el consumo eléctrico en función de los animales presentes y la producción de leche (medios de producción y producto final de estos sistemas ganaderos). Así, el consumo eléctrico global para las explotaciones auditadas en este estudio se sitúa en los $516( \pm 34,2) \mathrm{kWh}$ por vaca productora y año o $51( \pm 4,2) \mathrm{kWh}$ por cada $1000 \mathrm{~kg}$ de leche producida y año. En el presente estudio, el consumo eléctrico va-

Tabla II. Consumo eléctrico estimado (en $k W h$ y euros/año) de los principales procesos en una explotación de ganado vacuno lechero. (Estimated electricity consumption (in $\mathrm{kWh}$ and euros/year) of the main processes in a dairy cattle farm).

\begin{tabular}{lcccc}
\hline & \multicolumn{2}{c}{ kWh/año } & \multicolumn{2}{c}{ euros/año } \\
& media & e.e.m. & media & e.e.m. \\
\hline $\begin{array}{l}\text { Ordeño } \\
\text { Limpieza/ }\end{array}$ & 9183 & 1214,2 & 1194 & 157,8 \\
desinfección & 4695 & 2174,6 & 610 & 283,6 \\
Enfriamiento leche 6030 & 606,2 & 784 & 78,8 \\
\hline
\end{tabular}




\section{BARTOLOMÉ, POSADO, BODAS, TABERNERO DE PAZ, GARCÍA Y OLMEDO}

rió en función del tamaño de las explotaciones, mostrando las de tamaño medio (entre 200 y 400 mil kg de producción anual) los menores consumos (tabla III), si bien las diferencias no alcanzaron el nivel de significación requerido $(\mathrm{p}<0,05)$. Sin embargo, las explotaciones con mayor nivel de producción sí mostraron consumos significativamente mayores $(\mathrm{p}<0,001)$ en enfriamiento de la leche, bomba de vacío (procesos de ordeño), iluminación y otros conceptos con respecto a los otros dos grupos de granjas (<400000 kg leche/año; tabla III).

El consumo eléctrico de las granjas de producción de leche varía considerablemente en función de su tamaño, el tipo de sala de ordeño o el sistema de producción empleado (alimentación, distribución del alimento, tipo de estabulación). En el presente estudio, las explotaciones auditadas fueron clasificadas en función del tipo de sala de ordeño que poseían para obtener unos valores medios de consumo, observándose que en 5 explotaciones había robot de ordeño, en otras 5 sala rotativa y en 48 (un $60 \%$ del total de explotaciones) había una sala en espina de pescado, entre otros tipos de salas menos frecuentes (paralelo, circuitos, rts, tándem). Si bien el número de explotaciones de uno y otro tipo no es similar, trata de ser representativo del total de explotaciones presentes en la Comunidad. En cualquier caso, la media de consumo eléctrico diario de los procesos de ordeño (bomba de vacío + bomba de leche) de las explotaciones con sala en espina de pescado $(7 \mathrm{kWh})$ fue inferior al registrado para las explotaciones con sala rotativa ( $26 \mathrm{kWh})$ o con robot de ordeño $(24 \mathrm{kWh})(\mathrm{p}<0,001$; figura 2$)$. El consumo eléctrico que representan tanto el robot de ordeño como la sala rotativa es muy superior al de las explotaciones con

Tabla III. Comparación del consumo eléctrico y del coste asociado en las granjas de ganado vacuno lechero de Castilla y León en función de la producción anual de leche. (Comparison of electricity consumption and the cost associated in dairy cattle farms of Castilla y León depending on annual milk production).

\begin{tabular}{|c|c|c|c|c|c|}
\hline & \multicolumn{3}{|c|}{ Producción anual (kg) } & \multirow[b]{2}{*}{ d.e.r. } & \multirow[b]{2}{*}{$p$} \\
\hline & $<200000$ & $200000-400000$ & $>400000$ & & \\
\hline \multicolumn{6}{|l|}{ Consumo (kWh/año) } \\
\hline Tanque de frío & $2521^{\mathrm{a}}$ & $3025^{a}$ & $9262^{b}$ & 3839,7 & $* * *$ \\
\hline Bomba de vacío & $4414^{a}$ & $3438^{a}$ & $14153^{b}$ & 9064,3 & $\star \star \star *$ \\
\hline lluminación & $573^{a}$ & $454^{a}$ & $1898^{b}$ & 1524,5 & $* * *$ \\
\hline Ventilación & 473 & 282 & 1894 & 1373,9 & n.s. \\
\hline Termo eléctrico & 690 & 1066 & 1108 & 438,3 & n.s. \\
\hline Cintas de limpieza & 1322 & 471 & 7877 & 20750,9 & n.s. \\
\hline Otros & $337^{a}$ & $570^{a}$ & $4803^{b}$ & 3088,8 & $* * *$ \\
\hline \multicolumn{6}{|l|}{ Consumo medio } \\
\hline kWh/animal mayor de 24 meses & 477 & 464 & 566 & 303,6 & n.s. \\
\hline $\mathrm{kWh} / 1000 \mathrm{~kg}$ de leche producidos & 58 & 48 & 52 & 37,6 & n.s. \\
\hline \multicolumn{6}{|l|}{ Coste (euros/año) } \\
\hline Enfriamiento de leche & $328^{a}$ & $393^{a}$ & $1204^{b}$ & 499,2 & *** \\
\hline Ordeño & $585^{\mathrm{a}}$ & $469^{a}$ & $1872^{b}$ & 1194,6 & *** \\
\hline Limpieza y desinfección & 218 & 137 & 1015 & 2358,8 & n.s. \\
\hline
\end{tabular}

Archivos de zootecnia vol. 62, núm. 239, p. 452. 


\section{CONSUMO ELÉCTRICO EN GRANJAS DE VACUNO LECHERO}

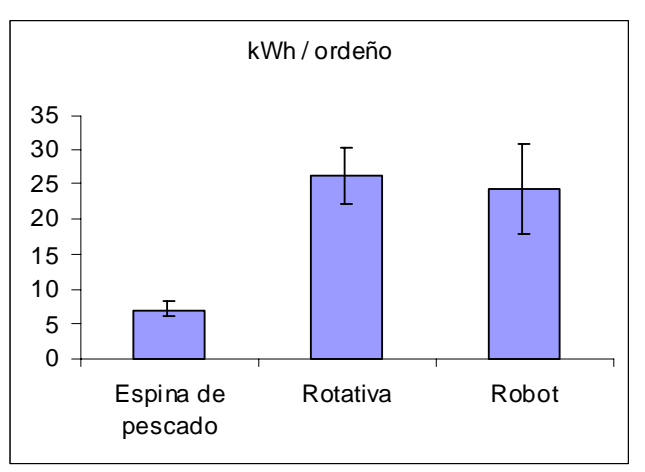

Figura 2. Consumo eléctrico por ordeño en función del tipo de sala de ordeño presente en la explotación. (Milking electricity consumption according to the milking system).

espina de pescado, lo que concuerda con los resultados observados por otros autores (Dollé, 2007; Murgia et al., 2008).

Por otro lado, en los países donde se han realizado estudios de este tipo se proporciona un amplio rango de datos; por ejemplo el consumo eléctrico anual estimado por vaca en Estados Unidos oscila entre los 700 y $1200 \mathrm{kWh}$, dependiendo de los estudios (Ludington y Johnson, 2003; WDATCP,
2006), en Irlanda es de alrededor de $300 \mathrm{kWh}$ (Upton et al., 2010), en Nueva Zelanda es de $163 \mathrm{kWh}$ (Hartman y Sims, 2006), en Italia de 466 kWh (Murgia et al., 2008), en el Reino Unido de 330 (Trimble, 2009) y en Francia de 470 kWh (Dollé, 2007; Béguin et al., 2008). Los datos del presente estudio se encuentran situados dentro de este rango, ocupando una posición intermedia.

Por otra parte, en un estudio realizado en Canadá se calculó que el consumo medio de electricidad por cada 1000 litros de leche producida se situaba en los $88,3 \mathrm{kWh}$ (NSAC, 2011); en el Reino Unido y en Italia este valor es ligeramente inferior, alrededor de $50 \mathrm{kWh} / 1000$ litros de leche (Trimble, 2009; Murgia et al., 2008), mientras que en Francia se sitúa entre los 60 y los $75 \mathrm{kWh} /$ 1000 L de leche (Béguin et al., 2008). Los resultados observados para las granjas de Castilla y León auditadas son semejantes a los publicados para granjas de Italia o el Reino Unido.

La distribución del consumo eléctrico en función de los diferentes equipos, procesos y sistemas dentro de la granja también varía ampliamente entre explotaciones. Así, en la figura 3 se pone de manifiesto esta

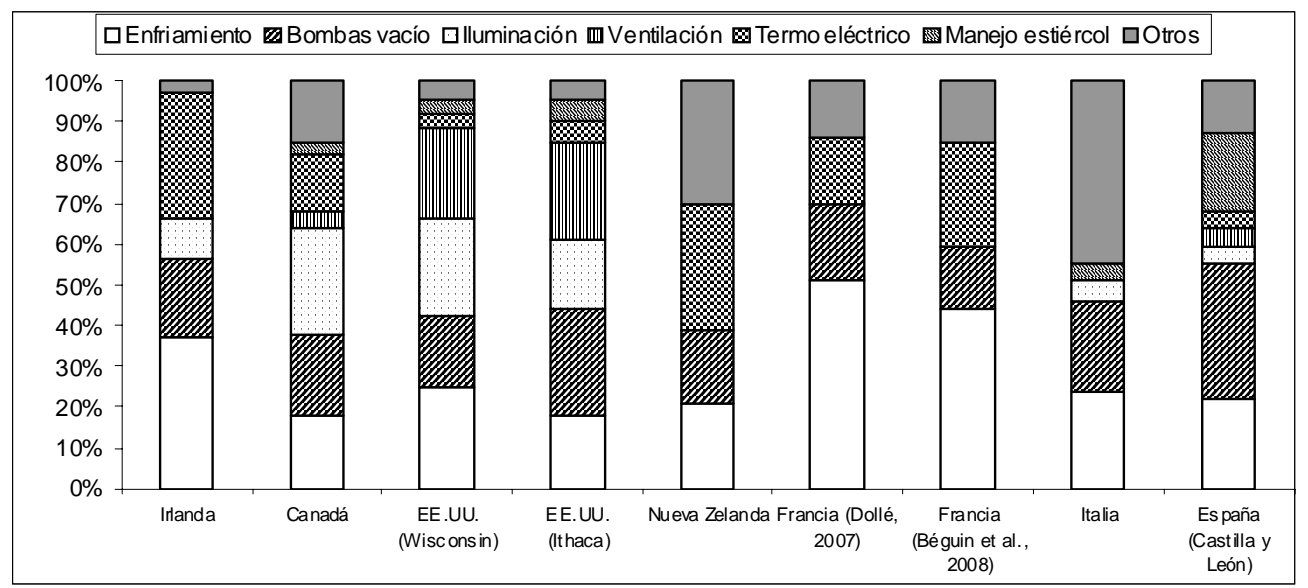

Figura 3. Distribución del consumo eléctrico (\%) entre diferentes sistemas y procesos en granjas de ganado vacuno lechero en diferentes localizaciones. (Distribution of electricity consumption (\%) between different systems and processes in dairy cattle farms in different countries). 


\section{BARTOLOMÉ, POSADO, BODAS, TABERNERO DE PAZ, GARCÍA Y OLMEDO}

variabilidad en distribución, mostrando tanto valores propios (España, Castilla y León) como datos recogidos en la bibliografía (incluso dentro de un mismo país varios estudios muestran diferencias en la distribución) para Irlanda (Upton et al., 2010), Canadá (NSAC, 2011), EE. UU. (Wisconsin: WDATCP, 2006; Ithaca: Ludington y Johnson, 2003), Nueva Zelanda (EECA 2009a,b), Francia (Béguin et al., 2008; Dollé, 2007) e Italia (Murgia et al., 2008). Como puede observarse, los procesos más variables son aquellos englobados bajo el epígrafe otros, puesto que incluye actividades muy heterogéneas cuya presencia o ausencia determina grandes cambios en el consumo (cintas de alimentación, bombeo de agua, mezcladores o molinos...). Los procesos de enfriamiento y de ordeño (bombas de vacío) representan siempre un porcentaje relativamente elevado, mientras que otros procesos, como la iluminación, varían considerablemente de unos estudios a otros, al igual que el manejo del estiércol (no considerado en algunos de ellos, como por ejemplo los de Francia y Nueva Zelanda). La variabilidad asociada al termo eléctrico o a la ventilación viene explicada en gran medida por la presencia o ausencia de estos elementos en función de las características de las explotaciones.

\section{BIBLIOGRAFÍA}

Béguin, E.; Caillaud, D.; Dollé, J.B.; Capitain, M.; Chambaut, H. et Charroin, T. 2008. Les consommation d'énergie dans les systèmes bovins laitiers, repères de consommation et pistes d'économie. Institut de l'Élevage. http:// www.inst-elevage.asso.fr/IMG/pdf_190850 023-v.pdf (22/05/2012). 32 pp.

Dollé, J.B. 2007. Les consommations d'énergie en bâtiments d'élevage bovin. Note de synthèse. Institut de l'Élevage. http://www.inst-elevage. asso.fr/IMG/pdf_CR_050733014.pdf (22/05/ 2012). $138 \mathrm{pp}$.

Edens, W.C.; Pordesimo, L.O.; Wilhelm, L.R. and Burns, R.T. 2003. Energy use analysis of major

\section{CONCLUSIONES}

Es posible la optimización del consumo eléctrico y la adopción de medidas de ahorro y eficiencia energética en las explotaciones de ganado vacuno lechero. Sin embargo, la implementación de medidas pasa necesariamente por la evaluación del consumo real producido en cada equipo y proceso de la explotación. Solamente así podrán considerarse de forma correcta las medidas de ahorro a tomar. En este sentido, la plataforma en línea (www.enerlacteo.com) posibilita que, de forma gratuita, los ganaderos puedan realizar una auditoría energética propia introduciendo las características y los datos de consumo de su explotación. A partir de la información proporcionada, esta herramienta web genera un informe con los índices de consumo y una serie de recomendaciones para tratar de mejorar la eficiencia energética de la explotación.

\section{AGRADECIMIENTOS}

Este trabajo ha sido realizado gracias a un proyecto (DER 20101899960100166) financiado conjuntamente por el Ministerio de Medio Ambiente y Medio Rural y Marino (MARM) y por el Fondo Europeo Agrícola de Desarrollo Rural (FEADER): Europa invierte en las zonas rurales.

milking center components at a dairy experiment station. Appl Eng Agric, 19: 711-716.

EECA. 2009a. Improving energy efficiency. A guide for New Zealand dairy farms. Energy Efficiency and Conservation Authority. http:// www.eeca.govt.nz/sites/all/files/dairy-farmenergy-efficiency-guide-march-09.pdf (22/05/ 2012). Wellington. New Zealand. 24 pp.

EECA. 2009b. Dairy farmers milk free energy. Energy Efficiency and Conservation Authority. https://www.eeca.govt.nz/sites/all/files/dairyfarmers-milk-free-energy-june-09.pdf (22/05/ 2012). Wellington. New Zealand. 8 pp. Hartman, K. and Sims, R.E.H. 2006. Saving energy 


\section{CONSUMO ELÉCTRICO EN GRANJAS DE VACUNO LECHERO}

on the dairy farm makes good sense. Proceedings of the $4^{\text {th }}$ Dairy Conference held at Hamilton New Zealand. Centre for Professional Development and Conferences. Massey University. http://www.dairynz.co.nz/file/fileid/ 39187 (22/05/2012). Palmerston North. New Zealand. pp. 11-22.

IDAE. 2010. Auditorías energéticas en instalaciones ganaderas. Parte 1: Manual para la realización de auditorías energéticas en instalaciones ganaderas. Serie: Ahorro y Eficiencia Energética en la Agricultura. Instituto para la Diversificación y Ahorro de la Energía. Madrid. 83 pp.

INE. 2002. Censo agrario 1999. Instituto Nacional de Estadística. http://www.ine.es/inebmenu/ mnu_agricultura.htm (28/05/2012).

INE. 2012. Censo agrario 2009. Instituto Nacional de Estadística. http://www.ine.es/inebmenu/ mnu_agricultura.htm (28/05/2012).

Jimeno, V. y Castro, T. 2009. El sector vacuno de leche y el seguro agrario en España. Ministerio de Agricultura, Alimentación y Medio Ambiente. http://aplicaciones.magrama.es/documentos_ pwe/seminarios/vacuno_leche_upm_ucm.pdf (28/05/2012).

Ludington, D. and Johnson, E.L. 2003. Dairy farm energy audit summary report. New York State Energy Research and Development Authority. http://www.nyserda.ny.gov/ /media/Files/ Publications/Energy\%20Audit\%20Reports/ dairy-farm-energy.ashx (22/05/2012). 25 pp.

MARM. 2010. Anuario de Estadística Agraria y Agroalimentaria. Ministerio de Medio Ambiente y Medio Rural y Marino. http://www.magrama. gob.es/estadistica/pags/anuario/2010/ae_ 2010_avance.pdf (03/05/2012). 1219 pp.

Murgia, L.; Caria, M. and Pazzona, A. 2008. Energy use and management in dairy farms. In: Innovation technology to empower safety, health and welfare in agriculture and agro-food systems. International conference. http://www. ragusashwa.it/CD_2008/lavori/TOPIC9/orale/ MURGIA.pdf (22/05/2012). September 15-17. Ragusa-Italy.

NSAC. 2011. Farm Energy Booklet Series, \#2 Dairy Farms. Nova Scotia Agricultural College. http:/ /nsac.ca/fens/factsheets/fens_energy booklet_2_dairy.pdf (22/05/2012).

Trimble, D. 2009. Report of pilot energy benchmarking project 2007/2008. College of Agriculture, Food and Rural Enterprise (CAFRE) Cuts Carbon. http://www.dardni.gov.uk/ruralni/ dairy_energy_report.pdf (25/05/2012). 6 pp.

Upton, J.; Murphy, M.; French, P. and Dillon, P. 2010. Dairy farm energy consumption. In: Dairying: Entering a decade of opportuniy. Teagasc National Dairy Conference 2010. Teagasc, Agriculture and Food Development Authority. http://www.teagasc.ie/publications/ 2010/52/52_NationalDairyConf2010Procee dings.pdf (22/05/2012). pp. 87-97.

WDATCP. 2006. Dairy farm energy management guide. Wisconsin Dept. of Agriculture, Trade and Consumer Protection. http://www.wista tedocuments.org/cdm/singleitem/collection/ p267601coll4/id/298 (22/05/2012). 51 pp. 